\title{
Effect of the Task, Visual and Semantic Context on Word Target Detection
}

\author{
Laure Léger $^{1}$, Charles Tijus ${ }^{1}$, and Thierry Baccino ${ }^{2}$ \\ ${ }^{1}$ Laboratoire Cognition \& Usages, Université de Paris VIII, \\ 2 rue de la Liberté 93526, SAINT DENIS cedex, France \\ laure.leger@cognition-usages.org \\ charles.tijus@univ-paris8.fr \\ ${ }^{2}$ Laboratoire Psychologie Expérimentale Quantitative, LPEQ, EA 1189, \\ Université de Nice Sophia Antipolis, \\ 24, Avenue des Diables Bleus 06357 NICE, cedex 04 France \\ baccino@unice.fr
}

\begin{abstract}
Although being a daily task, the search for a word among others words is a new research domain we investigated in order to find the kinds contextual factors that can facilitate semantic oriented visual search. We report two experiments assessing task context, visual context and semantic context. Some of our results are found to be those of classical non-semantic visual search, while others show the impact of the semantic context. Basic recommendations can be find out for Human-Computer conception and cognitive chronometry methodology.
\end{abstract}

\section{Introduction}

This article is about the factors that could facilitate the detection of a word among others. There is a practical question if we want to facilitate the rapid and successful finding by a user of the information s/he is looking for when facing Web sites. For instance, if you search the schedule of a film on a cinema web site, how must be semantic and visual information arranged in order to facilitate the visual search activity. But there is also a theoretical question about how much semantic features facilitate visual search. Studies of visual search conducted in cognitive psychology about attention and visual processes consist to show the participant a visual scene composed of no semantic visual stimuli such as letters, digits, squares, circles, triangles, ... The task of the participant is then to detect a particular target among others stimuli. This target can be well defined (to find the square) or can be ill defined (to find the intruder) as in oddity search task.

Treisman and her colleagues ([1] and [2]) had tested the effect of different visual features (such as color, orientation, form, ) on target detection efficiency. With a paradigm that consists to vary the number of the stimuli surrounding the target, they distinguished two visual search processes: a parallel search and a serial search. According to Treisman and Gelade [1] and Treisman and Gormican [2], the features (color, orientation, form...) of objects are first processed automatically and in 
parallel. Next stage following this parallel processing is to build the unicity of the object with focal attention on localization: the different features that are at the same localization are conjoined to form a unitary object, with sometimes illusory conjunction of features. If the object the participant is looking for (a red X) has a distinctive feature (red) in such a way this feature is in isolation (a red X among blue $\mathrm{Xs}$ ), then this target will be found during the parallel processing stage: the feature characterizing the target stimulus is detect pre-attentively and then «calls » attention to the position of the target stimulus in the visual field at the next stage. On the opposite, if the target can be distinguished from the process of a conjunction of features (a red X among red Os and blue Xs) then a serial search is applied at the second stage and consists inspecting the background items that form the context, one by one, until the target is found or until the participant decides that the target is absent. The number of non-target stimuli on the visual array has no effect on time detection of a target being distinguished by a single feature from the non-target stimuli: the target captures directly attention and this phenomena produces what is called "pop out". When the target is differentiated by a conjunction of features, in the serial search stage, the response time is dependent of the number of non-target stimuli: when the number of items in the context increases the time spent to detect the target increases because it is then necessary to conjoin the features (color and form for the above example) and to scan the whole scene, item by item, to find the target.

Efficiency of the visual search appears to be strongly dependant on the similarity between the target and its context (its background composed of others stimuli). According to Duncan and Humphreys [3], the more the target is similar to its context, such as a conjunctive target, the target sharing one attribute with one kind of context stimuli and one other attribute with a second kind of stimuli, the more difficult is its detection. Targets "pop outs" in case of large dissimilarity. Neisser [4], for instance, observed that it is easier to detect a $\mathrm{V}$ among round letters such as $\mathrm{O}, \mathrm{P}, \mathrm{D}, \mathrm{G}$ than among angular letters such as N, L, M, X.

Many factors could influence similarity between the target and the contextual nontargets and influence visual search. Sharing visual properties with the objects of the context is one of the factors that bring similarity. Another factor might be the nature of the shared or distinctive features. For instance, perceptual features might not be equivalent for the target detection. Treisman \& Gormican [2] find that when the target is to be in a pop out situation (the only item with a particular attribute), a deviant attribute (such as magenta for the color dimension) allows detecting the target more rapidly than a standard attribute (such as red). For these authors, this difference is due to a difference of activation between these two types of attributes: a deviant attribute produces more activation than a standard attribute. However, when the target is a feature conjunction (identifying a red X among red Os and blue Xs), a target sharing standard attributes is detected more rapidly than a target sharing deviant attributes [5].

A third factor, studied in the literature, is the proportion of each kind of stimuli in the context when the target is defined by a conjunction of properties shared with the context objects. This ratio is generally fifty-fifty: as much red Os and blue Xs to detect a red X. Poisson and Wilkinson [6] and Shen, Reingold and Pomplum [7] had shown that response time to detect a conjunctive target depends on the ratio of the two kind of non-target stimuli: target detection is facilitated when the two categories of stimuli that form the context do not appear in equal number in the visual display; as the number of each category approach equivalence, response time increases. 
A fourth factor is the semantic similarity between the target and the non-targets in its context. White [8] showed that semantic categories could play a facilitative role in target detection; thus detecting the letter " $\mathrm{O}$ " is easier when surrounded by numbers rather than surrounded letters. In the same way, it is easier to detect the number " $O$ " (zero) from a display of letters rather than a display of numbers. White's study was of interest because the semantic category was under the experimenter's control; the form of the target $(\mathrm{O})$ remained constant yet its meaning changed from the letter " $\mathrm{O}$ " to the number "O". In other words, detection is made easier when the target belongs to a different semantic category than the non-target stimuli.

In the visual search literature, the purpose of different studies is to determine factors that could facilitate or disturb visual search. In these studies, the material is simple: geometric shapes (such as squares, circles, triangles, bars, ...), digits or letters. Our study is for determining the factors that facilitate or disturb the detection of a word surrounded by others words. We reasoned that visual similarity effects might compete with more complex kind of features such as the semantic properties we get when using words as materials for visual search. This is also an ecological study, since semantic properties of perceived words might influence detection of a word-target in situations such as scanning an index, a newspaper or a web page. Whenever a group of words is perceived, these words could be semantically classified allowing a semantically contrasted target to be distinguished from other stimuli.

Two experiments were conducted to study the visual and semantic discrimination of a word-target from its context made of other words. In the first experiment, we examined the effects of the task context, of the semantic context surrounding the target and of the number of stimuli simultaneously displayed.

The task context is defined through the knowledge the participant has about the identity of the target: they know or they don't know its super-ordinate category.

The semantic context effect is explored by varying the semantic distance between the target and the non-target stimuli. For Rips, Shoben \& Smith [9] the semantic distance means "that when the subset was used as the predicate noun, the memorial representations of the subject and predicate nouns (ROBIN and BIRD) were closer together in some underlying semantic structure than when the superset was used as the predicate noun". For example, ROBIN is more semantically distant to ANIMALS than BIRDS. But this definition doesn't allow comparing two concepts that aren't on the same axis. For example, we can't evaluate the distance between TOYS and VEGETABLES. Then, we define semantic distance by the approximate number of superordinate categories between the target category and the superordinate category of both the target and the non-targets. The higher is the number of categories necessary to identify the common super-ordinate category of two concepts, the longer is the semantic distance between these two categories. For example, for the categories FISHES and BIRDS, it is easy to find a super-ordinate category ANIMALS that is their direct super-ordinate category. So FISHES and BIRDS are closely semantically related together. In opposite, for the categories FISHES and MANUFACTURED TOOLS, it is more difficult to find a category super-ordinate. So for these two categories (FISHES and MANUFACTURED TOOLS), we evaluate that they are semantically distant.

The effect of the number of stimuli in the context is studied by increasing the number of non-target words around the target-word.

In the second experiment, we examined the effect of the number of non-target words sharing a visual attribute with the target, the kind of visual property and the 
semantic typicality of the target. The effect of the number of non-target words is studied by increasing the number of non-target words that have the same color (red or black) or the same font (italic or not) than the target. We contrasted color and font in order to examine the effect of the kind of visual properties. The effect of semantic typicality was studied by using typical exemplar vs. non-typical exemplar of a category as being the target-word. It is strongly accepted in cognitive psychology that all exemplars of a category aren't equivalent: some are more representative of the category than others. For example, "robin" and "sparrow" are more typical of birds than "ostrich" or "penguin".

\section{Experiment 1}

This experiment investigated the effect of the semantic and visual background context, and of the task context on word visual detection.

The effect of semantic context is investigated by varying the semantic relatedness between the words surrounding the target. According to White [8], semantic differentiation between the target and the non-target facilitates detection. We reasoned that the detection of a target that is semantically distant to non-target words should be easier (higher success rate and shorter response time) than the detection of a target that is semantically close to the non-target words.

The visual context is investigated by varying the number of words surrounding the target. Because a word is a more complex item than a simple geometric form, we reasoned that the search for a word among others words would be serial. So we expect that increasing the number of non-target words would increase response time to detect the target.

The task context is investigated by providing or not providing the participant the semantic category of the target ("is there a animal" or "is there a word different to others"). Treisman and Sato [5] observed that when the target label wasn't given (the consign being to detect the intruder, an oddity search task), search is more difficult than when it was provided the participant: the search became a serial search as indicated by increase in response time as a function of increasing the number of stimuli simultaneously displayed. So, we reasoned that not providing information about the target would weaken performance (lower success rate and longer response time) than when providing information (the target' category label).

\subsection{Method}

Participants. The 54 participants were first-degree cycle students recruited in the psychology department. They did participated to another experiment on visual search. They were native French speakers or well mastered in French.

Stimuli. The experiment is computer-driven (FRIDA software). Stimuli are French words from 15 categories: flowers, vegetables, fruits, fishes, birds, insects, containers, tools, weapons, musical instruments, professions, toys, vehicles, sports, trees. The number of stimuli simultaneously displayed is of 9,17 or 25 , randomly posited in a matrix of five rows and five columns. For half of the trials, the target is present. A word of the same category than the non-target words is used when the target is absent. 
When displayed among distractors (non-target words), the target is semantically distant to the non-target words for half of the trials and semantically close for other trials. Note that, although orthographic and phonologic similarities might play an important role, we didn't compute these factors, assusming that they should be counterbalanced across experimental variations. Something we will further control. The words-stimuli were written in Arial Police, in black and with a 16 points size on a white background. They appeared on a screen with 800x600 resolution.

Procedure. Participants are distributed either on the well-defined condition group or on the ill-defined condition group. The experiment starts with the instructions provided to the participant that present the type of task (detection of a word among others), the response modalities. In addition, participants are asked quick answers without mistakes about if "yes" or "no" the target is present in the display. Before experimental trials, each participant makes 10 training trials.

A trial is searching for a word, the participant being instructed as follows: "Is there an exemplar of (semantic category (i.e. animal))?" for the well-defined target condition and "Is there a word different than others?" for the ill-defined target condition. When the participant has read and understood the question, s/he has to press the space key that makes the words being displayed. When the participant finds the target, s/he has to press the " $m$ " key, then to enter the name of the target with the keystroke. If the participant does not find any target, s/he has to press the "q" key. There were 72 experimental trials, for which 72 sets of 9,17 or 25 words were randomly displayed. Recorded data for each of the 54 participants are, for each of the 72 trials, the yes/no response, the word typed on the keyboard in case of Yes response, and the response time.

Experimental Design. $\mathrm{S}_{24}<\mathrm{C}_{2}>* \mathrm{D}_{2} * \mathrm{~N}_{3}$ where $\mathrm{S}_{24}$ corresponds to 24 participants per group: $\mathrm{C}_{2}$ (well-defined target versus ill-defined target), $\mathrm{D}_{2}$ corresponds to the two semantic distance between the target and the stimuli (close versus distant) and $\mathrm{N}_{3}$ correspond to the 3 size of non-target contextual stimuli: 8,16 or 24 stimuli.

Analysis. Positive trials (target is present) were retained for analysis. Success rate was computed by averaging the number of hits (to press " $\mathrm{m}$ " key and to give the right word) over the number of corresponding trials. Response time was computed only for hits. One participant of the well-defined target group and two participants of the illdefined target group have their data suppressed for the response time analysis because they did not get at least one hit for each of the 6 experimental conditions. Thus, ANOVA analysis of success rate was made on 27 participants per group and analysis of response time was made on 26 participants for well-defined target and of 25 participants for ill-defined target.

\subsection{Results and Interpretation}

First, the type of task had no significant effect on success rate (well-defined: mean: .83 , SD: .22, ill-defined: mean: .79, $S D: .23 ; \mathrm{F}(1,52)=1,25 ; \mathrm{p}=.27$, ns). However, as predicted an ill-defined target (mean: 7.92, SD: 3.71) is detected with longer response-time $(\mathrm{F}(1,49)=47,94 ; \mathrm{p}<.01)$ than a well-defined target (mean: 4.36; $S D: 1.8)$. Providing the participants the category of the target facilitates its detection. Second, a target that is semantically distant to the non-target words is detected with a 
higher success rate (mean $.87, S D: .18$ versus $.75 S D: .23 ; \mathrm{F}(1,42)=30,26, \mathrm{p}<.01$ ) and with a shorter response time (mean: $5.54, S D: 2.73$ versus mean 6.73, SD: 3.88; $\mathrm{F}(1,49)=51,64 ; \mathrm{p}<.01)$ than a target that is semantically close to the non-targets words. Semantic distinctiveness between the target and non-target words facilitates target detection. Third, the number non-target words had a significant effect on success rate $(\mathrm{F}(2,104)=19,99 ; \mathrm{p}<.01)$ and response time $(\mathrm{F}(1,52)=38,9 ; \mathrm{p}<.01)$. A target is detected with higher success rates when there are $8(\mathrm{M}: .87, S D: .19)$ and 16 non-target words (M: .84, SD: .2) than when there are 24 non-target words (M: .71, $S D$ : .26) $(\mathrm{F}(1,52)=38,8 ; \mathrm{p}<.01)$. Increasing the number of the non-target words in the context of the target increases significantly response time (8 stimuli : M: 4.70, SD: 2.04; 16 stimuli: M: 5.67, SD: 2.48 ; 24 stimuli: M: 8.04, SD: 4.33). As predicted, the less there is non-target words in the target context, the better its detection is.

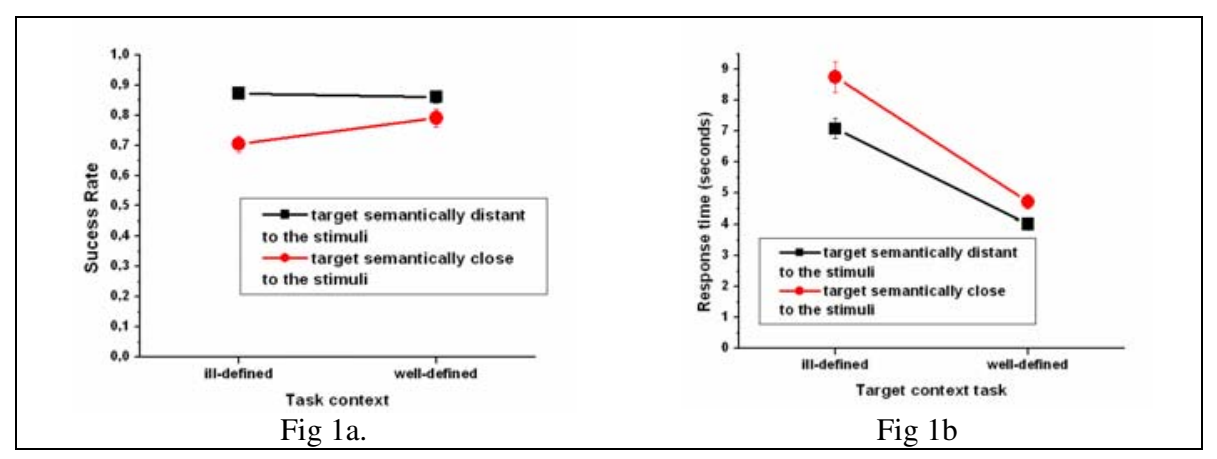

Fig. 1. Success rates (fig 1a) and response times (fig 1b) as a function of semantic context (close vs. distant) and of task context (ill defined versus well defined target)

The interaction between the task context (well vs. ill defined target) and the semantic context (close vs. distant) had a significant effect on success rate (fig. 1a, $F(1,52)=5,18$; $\mathrm{p}=.03$ ) and on response time (fig $1 \mathrm{~b}, \mathrm{~F}(1,49)=4,16 ; \mathrm{p}<.01$ ). As shown in the figure 1 , the difference between the two semantic distances is weaker when the target is well defined than when it is ill defined. So, providing the target its category label facilitates the detection of a target that is semantically close to the non-target words.

The interaction between the task context, the semantic distance context and the number of non-target words had no significant effect on success rate $(\mathrm{F}(2,104)=0,67$; $\mathrm{p}=.51$, ns) but a significant effect on response time $(\mathrm{F}(2,98)=3,23 ; \mathrm{p}=.04)$. The interaction between the semantic context and the task context had different effects as a function of the number of the non-target words. When there are 8 non-target words this interaction had no significant effect on response time $(\mathrm{F}(1,48)=0,05, \mathrm{p}=.82$, ns $)$. The effect of semantic context between the target and non-target words is the same whatever the task context. When there are 16 non-target words (figure $2 \mathrm{a}$ ), the effect of the semantic relatedness between the target and the non-target words depends on the task context $(\mathrm{F}(1,48)=7,44 ; \mathrm{p}<.01)$ : if the task is to detect the exemplar of a semantic category (well-defined target), response times are found shorter with targets that are semantically close than with targets that are semantically distant to the nontarget words. Such a difference is not observed when target are ill-defined. 


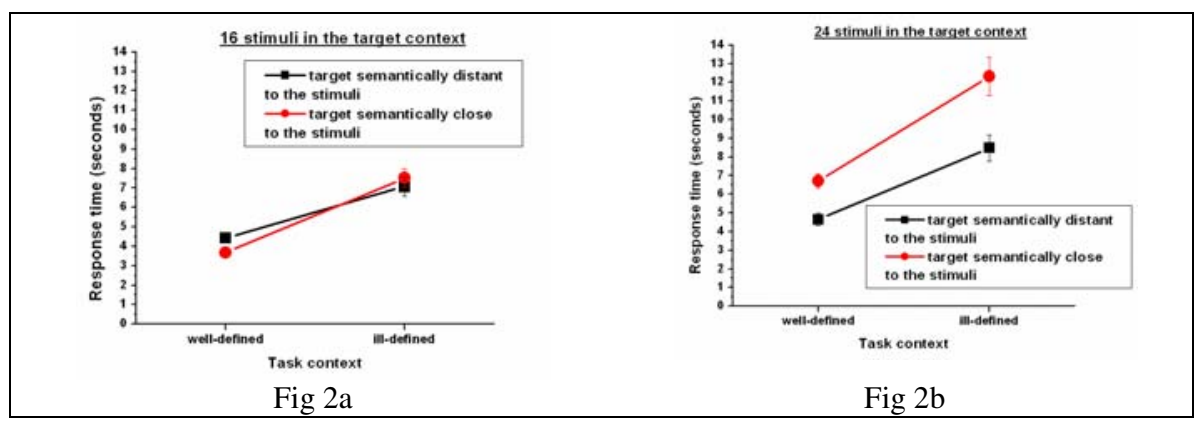

Fig. 2. Response time as a function of semantic context (close vs. distant) and of the task context (well vs. ill defined target) when the context is of 16 stimuli (fig 3a) and 24 stimuli (fig 3b)

When the context is of 24 non-target words (figure 2b), the interaction between the context task and the semantic context had an effect on response time $(F(1,48)=4,61$; $\mathrm{p}=.04)$ : the difference between the two semantic contexts is more important when the target is ill-defined than well-defined.

\subsection{Discussion and Conclusion}

In this first experiment, we showed at first that providing target label facilitates its detection. This result isn't very surprising: when the category target label is provided (well-defined task), participants have to map top down data (the target label) and the bottom up data (the words in the visual scene), processing each displayed word and comparing its category with the category label of the target. When the target label isn't provided (ill-defined task), participants in addition of processing each displayed word, have to find what relates all the displayed words (a common category), except one that is the target.

As we predicted, semantic distinctiveness plays a role on target detection: it's easier to detect a target semantically distant to non-target words than a target semantically closed to them. There is also an effect of the number of non-target words: increasing the number of non-target words increases the participants' response time to detect the target.

Of interest is the result that semantic distinctiveness interacts with the task context (providing or not the category target label) and with the number of non-target words. It helps having higher success rate when the category target label is unknown (fig. 1.a) and reducing response times when there are many non-targets (fig. 2b). Thus, processing the semantic context appears to play a role in visual search for a word among other words when the target word is ill-defined and when the task is difficult due to a large number of candidate words.

In the following experiment, in addition of semantic context, we explored the effect of target typicality, another semantic factor, and the effect of the number of words sharing a visual attribute with the target, a no-semantic factor. To examine in details the effect of these factors, we used the eye movements' record technique with the assumption that semantic dissimilarity (having a target surrounded by semantically distant non-targets words) might decrease rejection times of non-target words. 


\section{Experiment 2}

Longer response time to detect a semantically close target word to non-target words (as found in experiment 1) could come to the process of rejecting the fixated word as not being the target. Eye tracking enables the evaluation of semantic requirements, since items that are more difficult to process involve longer fixation times [10]. Thus, we predicted that trials in which non-target words were closely semantic related to the target would produce longer fixation times than those where non-target words were distantly semantically related to the target. Longer fixation times on trials where nontarget words are closely semantically related to the target would demonstrate a greater level of difficulty for deciding to reject a fixated item as not being the target item.

All of the exemplars of a semantic category aren't equivalents: some objects are more typical of the semantic category than others: sparrow is a better bird than ostrich. In addition to semantic context, we reasoned that typicality factor might have an effect on detection performances. We predicted that a typical target is detected easier than a non-typical target (higher success rate and shorter response time). Difficulty to detect a non-typical target should come from a difficulty to access the target meaning, its super-ordinate category or, even if so, the participant might verify if another word in the background better corresponds to the target category. The first difficulty (difficulty to attribute the good category to the target) should generate weaker success rate. The second (background verification) should generate longer response times.

In experiment 1 , we observed that the number of non-target words, in the target context, influenced visual search time. In experiment 1 , all words were of same visual attributes: same font, same color, same size... Experiment 2 investigates the effect of the number of words sharing a visual attribute with the target when the number of non-target words is invariant. The rational of experiment 2 is as follows.

According to Poisson and Wilkinson [6] and Shen, Reingold and Pomplum [7], we reasoned that a target sharing a visual attribute with a small set of non-target words will be detected with shorter response times than a target sharing a visual attribute with a large set of non-target words. We predicted that increasing the number of words sharing a visual attribute with the target would increase response time to detect the target. In addition, for a conjunctive target (which have two attributes of the property: conjunctive or conjunctive), we predicted that the more the ratio tends to equivalence (fifty-fifty), the more response times would increase.

\subsection{Method}

Participants. 40 native French speakers capable of reading from a viewing distance of $0.50 \mathrm{~m}$ without needing spectacles or contact lenses were recruited.

Apparatus. The oculometer used to measure eye movements was a device using corneal reflection (ASL 5000 model). This technique involves illuminating the participant's eye using infrared light and collecting reflections from the cornea and pupil. The position of the eye in $\mathrm{x}$ and y coordinates is sampled every $20 \mathrm{~ms}$. Ocular fixation is defined using a minimum of five sampled points separated from each other 
by at least $0.5^{\circ}$ of visual angle. This apparatus also enables measurements for each trial of response time, number of fixations, average fixation time and saccadic amplitudes.

Trial presentations were generated and response time was measured using a microcomputer. Experimental stimuli were presented on a flat 21-inch monitor screen (resolution: 1280 x 1024 pixels; color: 32 bytes).

Stimuli. According to the visual dimension under investigation color (black vs. red) or shape (normal vs. italic shape), the display was made of either has 31 words with black or red color, or with normal or italic font. An additional word is a conjunctive word: half-red half-black (detection) for the color dimension or half-italic font halfnormal font (detection) for the shape dimension. Except the target, all words belong to the same semantic category (e.g. 31 words are kinds of birds). The target is a word is of another category (e.g., vegetables). To evaluate the target typicality, we collected from 70 voluntary participants exemplars of the 24 semantic categories we used: the same used in the experiment 1 and the followingd: animals, furniture, salt food, games, mammals, buildings, sweet foods, drinks, clothes. When less than 3 over 35 participants named an exemplar, it was coded as being "non-typical", when more than 10 over 35 participants named an exemplar, it was coded as being "typical".

For each visual dimension (shape font or color), five visual displays were built by varying the number of words with normal font (shape dimension) or of black word (color dimension): 1, 7, 16, 24, or 30. The others words were italic (shape dimension) or red (color dimension). For example if the display was of 7 black words, it also had 24 red words and one conjunction color word. The figure 4 shows the visual context when varying the shape dimension. Equivalent context for color dimension is build substituting italic words by red words.

For each dimensional visual display, the target was either in italic font, normal font, or half normal and half italic for the shape dimension. For the color dimension, the target was either red, black, or red and black color conjunction.

Words are in Arial font, in 14 points size. The position of the target was randomized, but counterbalanced across different areas designated by the columns and rows of the visual display.

Procedure. Each participant was seated comfortably at a viewing distance of $0.50 \mathrm{~m}$ from the monitor screen and the ocular camera, with her/his chin stabilized in a chin rest. The experimenter read out the instructions that informed the participant that her/his ocular movements would be recorded and that s/he should avoid any further head movements. The instructions also described the experimental task to be carried out: the category of the item to be detected will appear in the center of the screen, when you left-click using the mouse, a group of words will appear on the screen; your task is to find the target, as quickly as possible and without making mistakes; as soon as you have found it, press the key and say aloud which word you have found; if you cannot find it, say "no". Next the experimenter began calibrating the oculometer; this process used a calibration card composed of nine black colored dots on a white background. After calibration was complete, six practice trials were presented to the participant before the experimental trials began. 
On each trial, the participant clicked using the mouse and the category of the target to be detected was displayed at the center of the screen. When the participant clicked again, the 32 words appeared on the screen. As soon as s/he had detected the target, s/he clicked again and said aloud the detected word. The experimenter then recorded this word. If s/he could not detect the target, s/he clicked and then said "no".

The order of presentation of different trials for each of the different experimental conditions was randomized. Each participant made a total of 120 trials.

When all trials were over, the experimenter obtained the ocular data for each trial. Each block of data included "yes/no" response, and the word said in case of Yes response, response time (as determined by the participant's click), the number of fixations and mean fixation time.

Note that participants didn't know exactly what is the target: they didn't know its identity and its perceptual feature. They only knew its super-ordinate category.

Experimental Design. The experimental design was $S_{20}<G_{2}>* P_{5} * A_{3} * D_{2} * T_{2}$, where $S_{20}$ was the 20 participants per group $\left(\mathrm{G}_{2}: \mathrm{g} 1\right.$ : color dimension, g2: shape dimension), $\mathrm{P}_{5}$ was the number of words of the same visual attributes $(1,7,16,24$, or 30 ), where $A_{3}$ was the target visual feature (black, red, half black - half red conjunction for the color dimension, normal font, italic font, half normal - half italic conjunction for the shape property), where $\mathrm{D}_{2}$ was the semantic context (distant or close semantic distance between the target and the non-target words) and where $\mathrm{T}_{2}$ was the typicality degree of the target (typical versus non-typical).

Analyses. Data were analyzed using an ANOVA statistical test. Only when the target was detected (correct responses) were response times and ocular measurements included in the analysis. The mean fixation duration corresponds to the average time per fixation before the target selection (total fixation duration over the number of fixations).

\subsection{Results}

We report separately results for each independent group (visual dimension) since effects of visual dimensions differ.

Italic Font Group. Analysis for success rates was made upon 20 participants. Analysis for response time was made upon participants who had a minimum of one success per experimental condition: 19 participants.

As shown in figure 3a, whatever the type of the target, the increase of the number of words sharing a same font with the target didn't generate a decrease of performances (less success and longer response times).

Contrary to our predictions, when the varied visual dimension is shape, target detection isn't facilitated by the decrease of the number of non-target words sharing the same shape than the target. Thus, for shape, a disproportionate ratio of distractors doesn't facilitate word target detection.

Color Group. Four participants were eliminated from the response time analysis because they didn't have at least one success by experimental condition. So, the response time analysis was made on 16 participants. 


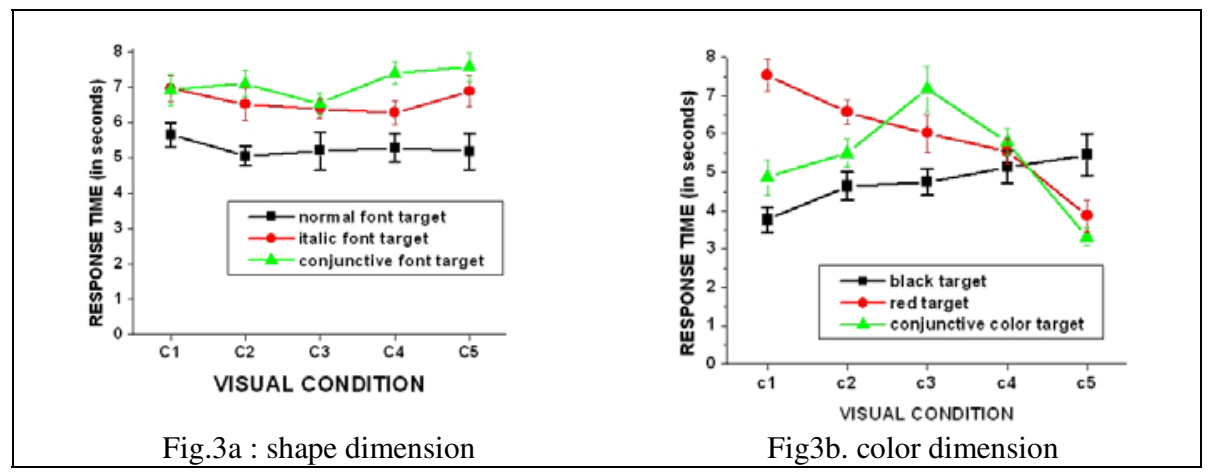

Fig. 3. Response times (in seconds) as a function of the shape of the target word (fig 3a) or the color of the target (fig. 3b) and of the number of non-targets words

As shown in figure $3 \mathrm{~b}$, an increase in the number of black words caused a success a response time increase $(\mathrm{F}(4,60)=3,15 ; \mathrm{p}=.02)$ to detect a black target and a response time decrease $(\mathrm{F}(4,60)=16,57 ; \mathrm{p}<.01)$ to detect a red target. For a conjunctive color target, a display with one black word provided lower success rates than others displays types $(\mathrm{F}(4,72)=7,60 ; \mathrm{p}<.01)$. On the other hand, the more the distractor ratio tended to equivalence and the more the response time increased $(F(4,60)=14,82$; $\mathrm{p}<.01)$. As we predicted, an increase in the number of non-target words sharing color with the target causes a reponse times increase. The detection of a conjunctive target was also facilitated by a disproportionate ratio of the two kinds of non-target words.

Typical targets were detected with higher success rates (mean .85 SD .17; $\mathrm{F}(1,18)=78.96, \mathrm{p}<.01$ ) and shorter response times (mean 4.49, SD 1.46; $\mathrm{F}(1,16)=34.09, \mathrm{p}<.01$ ) than non-typical targets (success rate : mean .66, SD .19; reponse time: mean 5.31, SD 1.99). As we predicted, detection of typical targets was found to be easier than detection of non-typical targets.

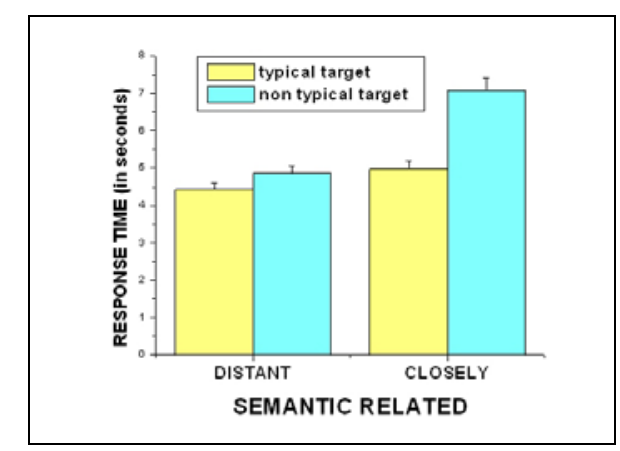

Fig. 4. Response times as a function of semantic context (distant vs. close) and of target typicality 
A target word being semantically distant to the non-target words was detected with higher success rates (mean 0.82, SD .17; F $(1,18)=66.03, \mathrm{p}<.01$ ) and with shorter response times (mean 4.33, SD 1.52; $\mathrm{F}(1,16)=46.37, \mathrm{p}<.01$ ) than a target being semantically close to the non-target words (success rate: mean $0.70, S D .21$; response time: mean 5.47, SD 1.86). This shorter response time was associated with a shorter mean fixation time (semantically distant : mean $431 \mathrm{~ms}, S D$ 64; semantically close: mean $458 \mathrm{~ms} S D$ 66; $\mathrm{F}(1,16)=16.66, \mathrm{p}<.01)$. According to our predictions, detection of a target was found to be easier when the target word is semantically distant to nontarget words rather than semantically close to non-target words.

Significant interaction effects were found for response time $(F(1,16)=43,25$, $\mathrm{p}<.01$ ). That is, participants took significantly more time and more fixations to detect a non-typical target surrounded by semantically close non-target words (see figure 4).

\subsection{Discussion and Conclusion}

Experiment 2 shows that to detect a word target, all visual features aren't equivalent. Varying the shape visual feature (normal font vs. italic font) produces no discrimination effect. This leads us to suppose that a shape feature doesn't allow discriminating the two groups of non-target words. Otherwise, for the color dimension, it seems that participants begin by glancing over the words group that have less representants before glancing over the words group which have more representants. Shen, Reingold and Pomplum [7] did find similar results. They observed that participants direct their first eye movements preferentially to the shorter distractors group before the larger distractors group. This search strategy is also used to detect a word cued by its superordinate category and for which the participant hadn't perceptual index. As we predicted, the semantic distance between the target and others words has an effect that is due to the facilitation or not to reject the fixated word as not being the target. As we predicted, target typicality plays also a role on target detection, but this is observed especially when the target word is semantically close to the non-target words.

\section{General Discussion and Conclusion}

Experiments 1 and 2 show that target's context influences detection. Here, the target context is made of 3 components: (i) the task - knowing or not knowing the category of the target -; (ii) the visual background of the display - the number of words surrounded the target, or the number of words sharing the same color than the target and (iii) the semantic relatedness between the target word and the non-target words the semantic distance between the target category and the category of the non-target words surrounded it -. Each of these components of the target context influences alone and in interaction the detection of a target word.

With the experiment 1 , we observed that target detection is easier when the participant knows in advance the target category. As discussed above, this result results from a direct mapping between the top down and bottom up data. We also observed that when the target is hard to detect some contextual properties could facilitate the detection. When the target word category is unknown or/and when it's 
surrounded by many of non-target words, having a semantically close target to the non-target words facilitates visual search and detection. When the participant knows the target category, decreasing the number of non-target words sharing the color with the target word facilitates also its detection. When the target is non-typical of its semantic category, providing a distant semantic context did facilitate its detection.

Using an oculometer, experiment 2, helps finding that these factors influence differently eye movements. The number of non-targets words sharing the target color, as well as the typicality of the target, influence the number of fixations, indicating a search difficulty, whereas the semantic distance between the target and the non-target words influences the mean fixation duration, indicating visual search difficulties.

In summary:

- Visual dimensions and visual attributes aren't equivalent for target detection [2].

- The number of words surrounded the target have an important effect on detection when the target is similar to the non-target words ([1], [3]) even if this similarity is semantic.

- The ratio of the two groups of non-target words influences target detection [6], [7].

- Semantic differentiation influences target detection [8].

- This search is a first step of studies we intend to pursuit in order to explore the effect of word properties on the detection of a lexical target. We find that the detection of target words among other words is guided by a serial search because it's necessary to inspect one by one each item (increasing the number of non-targets increase response time is what is expected fromserial search) and we identified some factors that could facilitate this one by one inspection.

This research also provides some basis for web interface ergonomics. A daily task is searching for information on the web. This search can be oriented either by precise information (search for the program of a cinema), or by a more global demand (search for information about horses). And in order to get this information, a user is facing several web pages that present words lists. This study shows that a word list presentation could respect some recommendations to facilitate the user's search, such as using visual discriminating features when there are some perceptual groups of words, taking few words by perceptual group, using words which have large semantic distance (don't belong to the same super-ordinate category) and using typical words.

This study is also about a mental chronometry in word visual search. It might be that in the first step, visual attributes of the visual scene (such as the number of red and black words) guide participant eye movements on objects that are of a high degree of distinctiveness. In a second step, the participant processes the fixated word: is it the target or not? And in a third step, either this word is the target and the search stops, or this word isn't the target and the eyes are moved towards another word that is distinctive from others and so on until the target-word is found or evaluated as being absent. This mental chronometry is similar to the guided visual search developed by Wolfe [11]. For Wolfe, the level of activation of each item of the background guides visual search: one by one item inspection from the item with higher activation to item with weaker activation. The activation value depends of the bottom up processes (resulting of the display appearance) and of the top down processes (resulting from the target knowledge such as its color, its identity, ...). We 
can assume a similar process when the participant doesn't know in advance what the target is going to be: the eye movements of the participant are leaded by perceptual attributes of the visual scene and semantic features are used for making decision.

Studies centered on the effects of contextual semantic features on visual search, such as experiments 1 and 2, could be complete what we already know about visual search.

\section{References}

1. Treisman, A. \& Gelade, G. (1980). A feature-integration theory of attention. Cognitive Psychology, 12, 97-136.

2. Treisman, A. \& Gormican, S. (1988). Feature analysis in early vision: Evidence from search asymetries, Psychological Review, 95, 15-48.

3. Duncan, J. et Humphreys, G.W. (1989). Visual search and stimulus similarity. Psychological Review, 96, 433-458.

4. Neisser, U. (1963). Decision time without reaction time: Experiments in visual scanning. American Journal of Psychology, 76, 376-385.

5. Treisman, A. et Sato, S (1990). Conjunction search revisited. Journal of Experimental Psychology: Human Perception and Performance,16, 459-478.

6. Poisson, M.E. et Wilkinson, F. (1992). Distractor ratio and grouping processes in visual conjunction search. Perception, 21, 21-38.

7. Shen, J., Reingold, E.M., \& Pomplum, M. (2000). Distractor ratio influences patterns of eye movements during visual search. Perception, 29, 241-250.

8. White, M.J. (1977). Identification and catégorization in visual search. Memory and Cognition, 5, 648-657.

9. Rips, L.J, Shoben, E.J. \& Smith, E.E. (1973). Semantic distance and the verification of semantic relations. Journal of verbal learning and verbal behavior, 12, 1-20.

10. Just, M.A. \& Carpenter, P.A. (1980). A theory of reading: From eye fixations to comprehension. Psychological Review, 87, 329-354.

11. Wolfe, J.M. (1994). Guided Search 2.0: A revisited model of visual search. Psychonomic Bulletin and Review, 1, 202-238. 